\title{
Irinotecan and oxaliplatin: an overview of the novel chemotherapeutic options for the treatment of advanced colorectal cancer
}

I. Grivicich ${ }^{1}$,

D.R.A. Mans ${ }^{1}$,

G.J. Peters ${ }^{2}$ and

G. Schwartsmann ${ }^{1}$
${ }^{1}$ South-American O ffice for Anticancer D rug Development (SOAD), Universidade Luterana do Brasil, Canoas, RS, Brasil

${ }^{2}$ Department of Medical O ncology, Free University Hospital, Amsterdam, The Netherlands

Correspondence
I. Grivicich
South-American Office for Anticancer
Drug Development (SO AD) - CINCAN
Universidade Luterana do Brasil
Rua Miguel Tostes, 101, Prédio 22
92420-280 Canoas, RS
Brasil
Fax: +55-51-478-1747
E-mail: ivanagrivicich@ zaz.com.br
........................

Received October 26, 2000

Accepted June 5, 2001

\section{Abstract}

Colorectal cancer is one of the most frequent malignancies in humans and an important cause of cancer death. Metastatic colorectal cancer remains incurable with available systemic therapeutic options. The most active cytotoxic drug against this malignancy, the antimetabolite 5-fluorouracil, was developed more than forty years ago, and as a single agent produces responses in only 10 to $15 \%$ of patients which in general last less than one year. Efforts to ameliorate these poor results resulted in the 5-fluorouracil/leucovorin combination, which enhances response rates about two-fold, without, however, significantly improving survival rates. The recent emergence of a handful of new 5fluorouracil analogues and folate antagonists, as well as the topoisomerase I inhibitor irinotecan, and the third-generation platinum compound oxaliplatin, is likely to alter this gloomy scenario. These agents are at least as effective as 5-fluorouracil in patients with advanced colorectal carcinoma, both untreated and previously treated with 5fluorouracil-based regimens. This has led to the approval of irinotecan as second-line treatment for 5-fluorouracil-refractory disease, while the use of oxaliplatin has been suggested for patients having a defective 5-fluorouracil catabolism. Recently, FDA approved the combination of irinotecan with 5-fluorouracil and leucovorin for first-line treatment of advanced colon cancer. Based on the synergistic preclinical antitumor effects of some of these agents, their meaningful singleagent activity, distinct mechanisms of cytotoxicity and resistance, and only partially overlapping toxicity profiles, effective combination regimens are now being developed, which are likely to lead to a new, more hopeful era for patients suffering from advanced colorectal carcinoma.

\section{Introduction}

Colorectal cancer is one of the most common human malignancies, and is among the leading causes of cancer death $(1,2)$. Incidence rates vary around the world, the high-

\section{Key words}

- Colorectal carcinoma

- 5-Fluorouracil

- Irinotecan

- Oxaliplatin est being observed in industrialized countries such as the USA, with 35.8 cases per 100,000 individuals, and the lowest in India, with 3.4 cases per 100,000 individuals (1). This suggests that the development of colorectal carcinoma is influenced by variations 
in environment and life style. This assumption is supported by the rapidly increasing incidence of colorectal carcinoma in individuals originating from low-incidence countries when they migrate to a high-incidence host country (1).

Incidence and mortality rates of colorectal cancer in the USA and Western Europe seem to have declined or remained constant over the past ten years (1). In Brazil, on the other hand, these figures seem to be increasing (2), as suggested by a recent report from the Instituto Nacional de Câncer (2), according to which colorectal carcinoma affects nowadays $30 \%$ more Brazilians than in 1989 , and kills approximately $40 \%$ more cancer patients in our country than ten years ago.

Presumed risk factors for colorectal carcinoma are meals poor in vegetables but rich in meat and animal fats, cigarette smoking, excessive alcohol consumption, limited physical activity, and low calcium intake (13 ). However, their relative importance is quite different and has been disputed in the literature (3). In addition, certain hereditary factors may predispose to colorectal carcinoma. Among these are familial adenomatous polyposis, the hereditary non-polyposis colorectal cancer syndromes, Gardner's syndrome, Turcot's syndrome, and Lynch's syndrome (3). Patients with inflammatory bowel diseases also run an increased risk for colorectal carcinoma (3).

In all cases, the development of colorectal carcinoma has been associated with the occurrence of sequential mutations in the cells of the intestinal epithelium (3). These include inactivation of the tumor suppressor gene APC (adenomatous polyposis coli), mutations in the ras oncogene, and inactivation of the tumor suppressor genes $D C C$ ('deleted in colon cancer') and p53. These mutations have been suggested to coincide with the occurrence of premalignant, hyperplastic lesions, the development of dysplasia, and the subsequent progression to carcinoma in situ and metastatic disease. How- ever, not all of these alterations may take place, and not necessarily in this order. Examples of alternative genetic changes associated with the development of colorectal carcinoma are aberrations in HER2/erb-B2 (which encodes for the epidermal growth factor receptor), c-myc (which encodes for a transcription factor), $M C C$ (which, like $D C C$, encodes for a cell adhesion protein), and $h M S H 2$ and $h M L H 1$ (which encode for proteins of the mismatch repair system) (3).

\section{Treatment options}

Cancer of the colon is a highly treatable and curable disease when localized. In these cases, surgery is the primary treatment option (3). The prognosis of patients following surgery depends on the tumor stage. In those patients with lymph node involvement, approximately $50 \%$ will eventually experience metastatic disease progression following surgery (3). Systemic chemotherapy, therefore, plays an important role in such patients. Unfortunately, cytotoxic drug therapy generally produces a partial clinical response of short duration, making this form of treatment only of palliative value (3).

\section{5-Fluorouracil}

The most commonly used protocols in advanced colorectal carcinoma include the fluorinated pyrimidine antimetabolite 5-fluorouracil, the relevant aspects of which are extensively described in Ref. 4. This drug was rationally synthesized in the 1950 s, following the observation that rat hepatomas preferentially utilized uracil when compared to normal tissues, suggesting the possibility of tumor-specific targeting of intracellular routes for uracil metabolism. As a single agent, 5-fluorouracil is only modestly active against advanced colorectal cancer, producing typical response rates of 10 to $15 \%$ which in general last less than one year $(3,4)$. However, because of the lack of more effective 
drugs against colorectal carcinoma, it is still the mainstay in the treatment of this disease.

In addition to being active against malignancies of the gastrointestinal tract, 5-fluorouracil is active against breast and head and neck carcinomas (4). The major toxic effects of 5-fluorouracil are mucositis, diarrhea, nausea and vomiting, and myelosuppression, which vary with dose, schedule, and route of administration $(3,4)$.

\section{Mechanism of action}

As shown in Figure 1, 5-fluorouracil is a prodrug that must be intracellularly converted into the active phosphates 5-fluorodeoxyuridine monophosphate (FdUMP), fluorouridine-5' -triphosphate (FUTP), and fluorodeoxyuridine-5'-triphosphate (FdUTP). The metabolic steps involved in these conversions are addressed in detail in Refs. 4 and 5 .

The main mechanism of 5-fluorouracil cytotoxicity probably involves interference of FdUMP with the activity of thymidylate synthase. Thymidylate synthase is a cytosolic enzyme that catalyzes the methylation of deoxyuridine-5'-monophosphate (dUMP) to yield deoxythymidine-5' -monophosphate (dTMP), a precursor of deoxythymidine triphosphate (dTTP), one of the deoxyribonucleotides required for DNA synthesis $(4,5)$. Intracellularly generated FdUMP associates covalently with thymidylate synthase and 5,10-methylene-tetrahydrofolate to form a stabilized ternary complex (6), leading to the inhibition of dTMP production and thus DNA synthesis (6).

Using breast and colon cancer cell lines, activity levels of thymidylate synthase were found to correlate well with the degree of 5fluorouracil cytotoxicity (6,7). Moreover, thymidylate synthase activity was significantly lower in tumors from colon cancer patients who responded to 5-fluorouracil when compared to those who did not respond (7). Thus, high thymidylate synthase activity levels are probably responsible for the resistance of tumor cells to 5-fluorouracil, and can predict the responses to treatment with this agent (7).

Although these data support the idea that inhibition of thymidylate synthase is the principal mechanism of 5-fluorouracil cytotoxicity, abnormal RNA and protein processing after incorporation of FUTP into various RNA species (8) and premature chain termination and/or DNA strand breakage upon incorporation of FdUTP into DNA (9) may also contribute to varying degrees of 5-fluorouracil cytotoxicity.

\section{Modulation of 5-fluorouracil}

The limited tumor responses to 5-fluorouracil prompted numerous efforts to improve the clinical efficacy of the drug. These

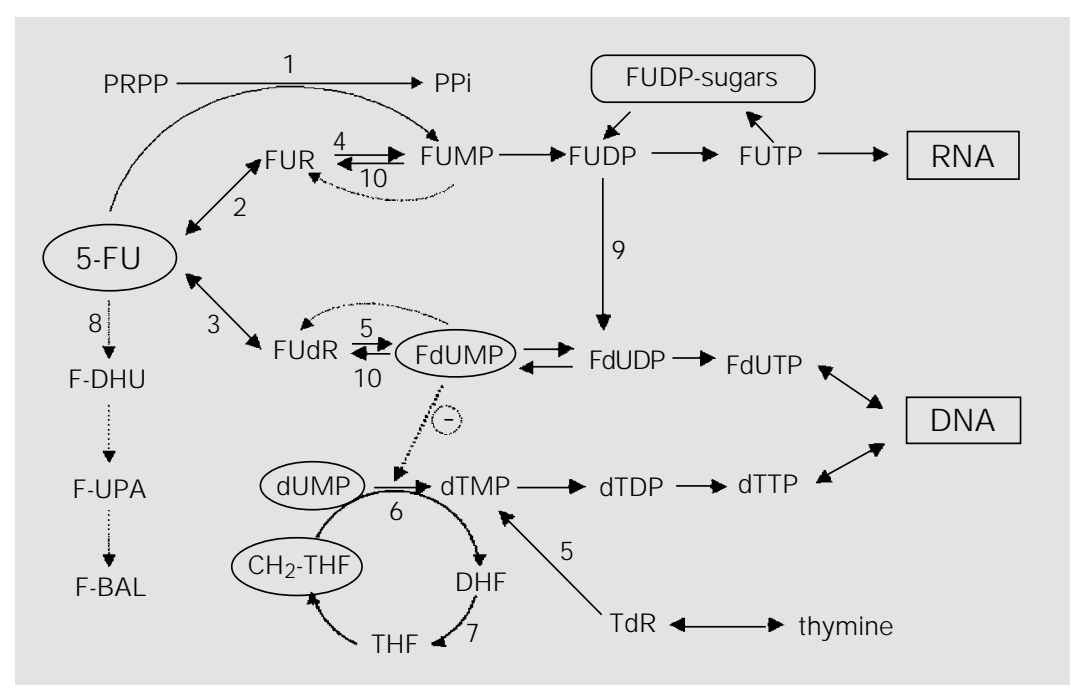

Figure 1. Metabolic pathways and mechanism of action of 5-fluorouracil (5-FU). Enzymes catalyzing these reactions are 1 , orotate phosphoribosyltransferase; 2 , uridine phosphorylase; 3 , thymidine phosphorylase; 4 , uridine kinase; 5, thymidine kinase; 6 , thymidylate synthase; 7, dihydrofolate reductase; 8 , dihydropyrimidine dehydrogenase; 9 , ribonucleotide reductase; 10, 5'-nucleotidases and phosphatases. FUMP, FUDP, FUTP: fluorouridine -5'-mono-, di-, and triphosphate, respectively; FdUMP, FdUDP, FdUTP: fluorodeoxyuridine5'-mono-, di-, and triphosphate, respectively; dUMP: deoxyuridine-5'-monophosphate; dTMP, dTDP, dTTP: deoxythymidine-5'-mono-, di-, and triphosphate, respectively; PRPP: 5phosphoribosyl-1-pyrophosphate; FUR: fluorouridine; FUdR: fluorodeoxyuridine; F-BAL: $\alpha$-fluoro-ß-alanine; F-UPA: fluoroureidopropionate; F-DHU: 5-fluorodihydrouracil; DHF: dihydrofolate; THF: tetrahydrofolate; TdR: thymidine; PPi: pyrophosphate. Modified from Ref. 61. 
included its administration by continuous infusion instead of bolus injection in order to increase dose intensity (10), and its local or regional administration, such as by direct hepatic arterial infusion for patients with liver metastases (11). Also, several combinations of 5-fluorouracil with other cytotoxic drugs have been evaluated, such as with methyl $\mathrm{CCNU}$, mitomycin $\mathrm{C}$, or cisplatin (reviewed in Refs. 5 and 12).

Furthermore, many attempts have been made to pharmacologically manipulate the intracellular metabolizing pathways of 5fluorouracil in order to selectively increase its antitumor efficacy and/or to decrease its toxicity (12). Examples are the use of the following substances: methotrexate or $\mathrm{N}$ phosphon-acetyl- $L$-aspartate (PALA) to increase 5-phosphoribosyl-1-pyrophosphate and thus the conversion of 5-fluorouracil to FUMP, interferons to reduce 5-fluorouracil plasma clearance, thus increasing drug tissue exposure and 5-fluorouracil-mediated DNA damage, uridine to prevent 5-fluorouracil incorporation into RNA and to reduce toxic side effects, thymidine to prevent 5fluorouracil degradation, prolonging 5-fluorouracil exposure, allopurinol to inhibit orotic acid accumulation, improving 5-fluorouracil activation, dipyridamole to inhibit 5-fluorodeoxyuridine efflux, thus increasing FdUMP retention, levamisole as an immunomodulator of 5-fluorouracil cytotoxicity, and leucovorin (5-formyltetrahydrofolate) to increase thymidylate synthase inhibition and retention (12).

Although these studies have provided valuable information about the mechanism of action and clinical pharmacodynamics of 5-fluorouracil, meta-analysis of all data showed meaningful benefit only by the addition of leucovorin to 5-fluorouracil (13). This combination can increase the response rates of colorectal carcinoma to 5-fluorouracil by approximately two-fold when compared to 5-fluorouracil alone. This is due to an enhanced inhibition of thymidylate syn- thase in the presence of excess leucovorin, due to the prolonged stabilization of the FdUMP-thymidylate synthase-folate ternary complex $(6,12)$. This finding provided the rationale for the current use of 5-fluorouracil plus leucovorin as first-line therapy in advanced colorectal carcinoma.

\section{Novel chemotherapeutic options}

Despite the greater clinical efficacy of 5fluorouracil plus leucovorin compared to 5fluorouracil alone, this combination did not lead to significant improvements in survival rates, and relapses continue to be the rule (12). Therefore, the search for more effective agents for the treatment of advanced 5fluorouracil-refractory colorectal carcinoma continued. This led to the development of some new 5-fluorouracil analogues and folate antagonists, which are currently under investigation.

Capecitabine or Xeloda ${ }^{\circledR}$ or $\mathrm{N}_{4}$-pentoxycarbonyl-5'-deoxy-5-fluorocytidine (reviewed in Ref. 14) is a rationally designed oral fluoropyrimidine carbamate that, after selective conversion to 5-fluorouracil within solid tumors, acts by inhibiting thymidylate synthase activity. This would theoretically yield two advantages, viz. enhanced drug concentrations at the tumor site and thus greater antitumor activity, and reduced drug levels in normal tissues with a consequent reduction in systemic toxicity. After displaying encouraging preclinical activity in vivo against colorectal carcinoma including 5fluorouracil-resistant tumors, capecitabine underwent clinical testing. In phase II studies involving diverse schedules with or without leucovorin, capecitabine was found to induce $21-24 \%$ objective responses in patients with untreated metastatic colorectal cancer, but less toxicity when compared to 5-fluorouracil/leucovorin. Since patients treated with intermittent capecitabine showed a longer time to disease progression when compared to the other schedules used, and 
also enjoyed a one-week rest period, this schedule was selected for phase III evaluation.

UFT is a combination of uracil and tegafur (1-[2-tetrahydrofuranyl]-5-FU, Ftorafur) in a fixed molar ratio of 4:1 (15). Tegafur is a prodrug that is hydroxylated and converted to 5-fluorouracil by microsomal P450 enzymes in the liver. In Japan this drug was developed as an oral formulation in combination with uracil in order to maximize its therapeutic selectivity. Uracil is a weak competitive inhibitor of dihydropyrimidine dehydrogenase, preventing degradation of 5fluorouracil by this cellular enzyme. In two multicenter, randomized, phase III trials involving patients with advanced colorectal cancer, the activity of UFT/leucovorin was equivalent to that of intravenous 5-fluorouracil/leucovorin but with a significantly lower major toxicity. The predominant side effect of UFT, diarrhea, is generally selflimited and easily managed. Myelosuppression and half-foot syndrome were rarely noted in these trials. Other combinations of UFT also demonstrated good activity. In a Japanese clinical study on gastric cancer, response rates of $37 \%$ were observed for the combination of UFT and doxorubicin, of $47 \%$ for UFT with doxorubicin and cisplatin, and of $40 \%$ for UFT with doxorubicin, cisplatin and etoposide. Ongoing trials are defining the roles of this agent (15).

Examples of new thymidylate synthaseinhibiting folate antagonists under development or registered (reviewed in Ref. 16), are AG337 (Thymitaq, Nolatrexed ${ }^{\circledR}$ ), AG331, LY231514 (ALIMTA, Pemetrexed ${ }^{\circledR}$ ), and ZD1694 (Tomudex ${ }^{\circledR}$, Raltitrexed). AG337 and AG331 are lipophilic antifolates that do not require activation by folylpolyglutamate synthetase (FPGS) and do not require transport into cells by the reduced folate carrier (RFC). This would yield the theoretical advantage of circumvention of resistance mechanisms involving low FPGS and/or low RFC expression. AG331 is essentially simi- lar to AG337, but has a greater potency in terms of thymidylate synthase inhibition. Both agents are under early clinical evaluation. In phase II studies, AG337 displayed modest activity in untreated head and neck cancer patients. LY231514 is a unique multitarget antifolate that produced encouraging responses (16\% overall response rate) in untreated patients with colorectal carcinoma in a phase II clinical trial. Of the newly available thymidylate synthase inhibitors, ZD1694 is the only one that has thus far advanced to phase III studies. This compound displayed activity against colorectal carcinoma comparable to that of 5-fluorouracil/leucovorin, while exhibiting a more favorable toxicity profile (16).

In addition to these developments, a number of novel chemotherapeutic agents, which proved to be effective against advanced colorectal carcinoma, have emerged. Among these are the topoisomerase I-inhibiting agent irinotecan and the platinum analogue oxaliplatin, which are described below in greater detail.

\section{Irinotecan}

Irinotecan (7-ethyl-10-(4-(1-piperidino)1-piperidino) carbonyloxy-camptothecin; CPT-11; Camptosar ${ }^{\circledR}$ ) is a semisynthetic, water-soluble derivative of camptothecin, an alkaloid isolated from the bark and wood of the Chinese tree Camptotheca acuminata (17). Camptothecin advanced to clinical trials in the 1970s (18), but despite some antitumor activity, its poor water solubility and unpredictable toxicity profile (diarrhea, cystitis, stomatitis, and myelosuppression) led to the termination of its further evaluation.

Renewed interest in camptothecin was prompted by the identification of topoisomerase I as its principal target in the 1980 s (19). The discovery of this 'novel mechanism of action' fueled efforts directed at the synthesis of more water-soluble analogues having a more favorable toxicity profile as 
Figure 2. Metabolic conversion of irinotecan into $\mathrm{SN}-38$. 1: carboxylesterase-converting enzyme. Modified from Ref. 62. well as consistent antitumor activity. In addition to irinotecan, several other promising analogues such as topotecan, 9-aminocamptothecin, and 9-nitrocamptothecin emerged from these efforts. These have been extensively reviewed in Ref. 20.

Irinotecan displays meaningful activity against colorectal carcinoma, both singly and in certain combinations, in first- as well as second-line studies (reviewed in Ref. 20). This compound was further found to be active against gastric and pancreatic carcinoma (21), cervical carcinoma, small cell and non-small cell lung carcinoma (22), highgrade central nervous system tumors (23), as well as leukemia and lymphoma (24).

\section{Mechanism of action}

Irinotecan has a piperino side chain at the C-10 position (Figure 2), which can be cleaved by carboxyl esterases that are present abundantly in the liver, but can also be found in other tissues and cells. This reaction yields the 100- to 1000 -fold more biologically active metabolite, 7-ethyl-10hydroxycamptothecin or SN-38 (25). Like camptothecin, both irinotecan and $\mathrm{SN}-38$ act by introducing single-strand DNA breaks upon the stabilization of DNA-topoisomerase
I complexes $(19,20)$. As shown in Figure 3, these are formed in duplicating DNA as intermediates of topoisomerase I-catalyzed DNA cleavage and religation reactions, necessary to relieve the torsical strain generated by advancing replication forks $(19,20)$.

The single-strand breaks themselves are not cytotoxic, because they are highly reversible and are rapidly repaired when the drug is removed. According to the 'fork collision model', lethal, irreversible DNA damage only occurs with ongoing DNA synthesis, i.e., when a replication fork encounters a cleavable complex, resulting in the formation of a complete double-strand DNA break $(19,20)$.

\section{Preclinical studies}

Initial preclinical studies with irinotecan are summarized in Ref. 24. The drug showed significant antitumor activity against a broad spectrum of tumor cell lines and xenografts, including breast, gastric, and colon cancer. Irinotecan was also active against multidrugresistant tumor cells expressing the $m d r$ gene, both in vitro and in vivo, indicating that it may be a poor substrate for cellular drugexporting mechanisms (24). The antitumor effects of irinotecan were found to be highly

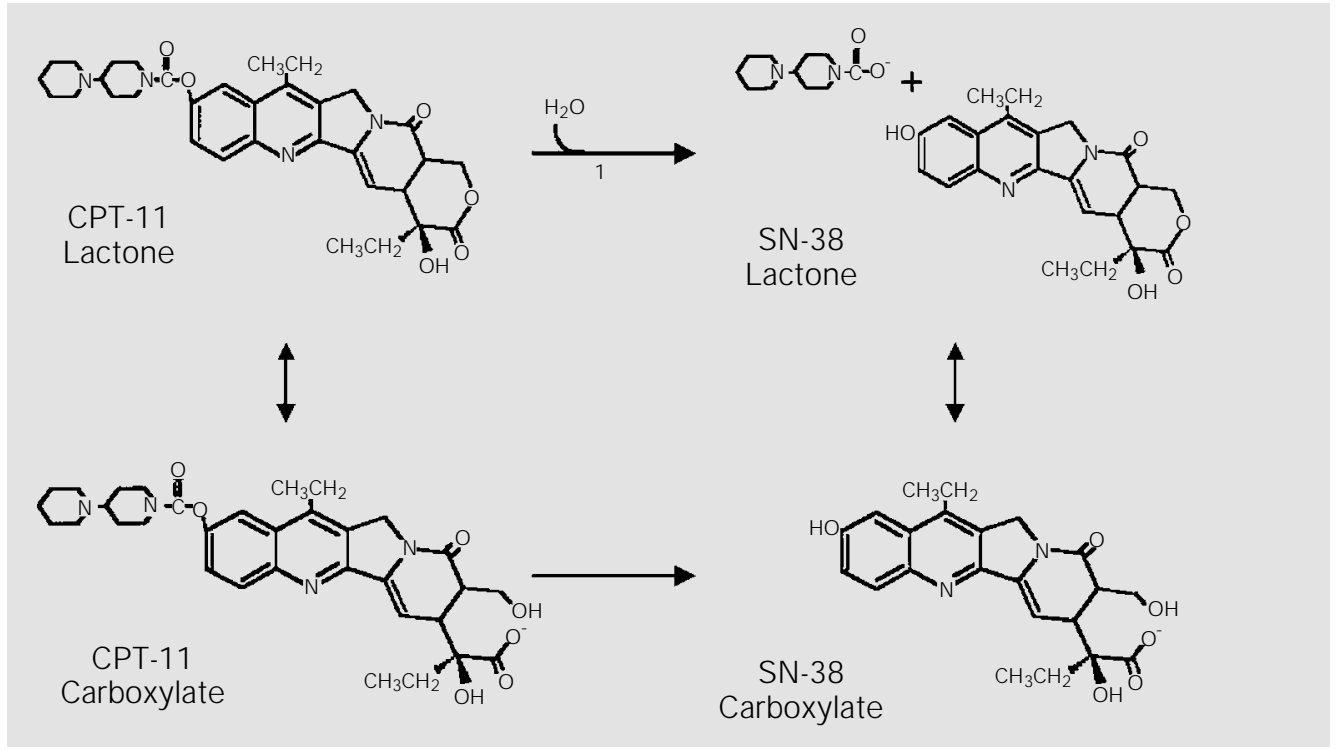


schedule-dependent in laboratory animals, with significant improvement by repeated administration of smaller doses for prolonged periods of time instead of higher doses at less frequent intervals (26). Studies to assess the definite clinical significance of this finding are still needed.

Preclinical studies of the irinotecan-5fluorouracil combination (see for instance Refs. 27-31) seemed to yield contradictory results in terms of the optimum sequence of drug administration. With virtually each combination and sequence, antagonistic, additive, and/or synergistic interactions were reported, which might be due to differences in experimental conditions. These include, among others, the choice of cell line, the use of a cell culture or a nude mouse-xenograft model, and the use of a short or a continuous period of drug exposure, conditions known to considerably influence the antiproliferative effects of both 5-fluorouracil and irinotecan. As an example, in a recent study from our laboratory with SW620, HT-29, and SNU-C4 human colon carcinoma cell lines (31), differences in intrinsic drug sensitivity and target enzyme activities appeared to partially determine the response to the 5-fluorouracil-irinotecan combination. Thus, prior administration of irinotecan at a fixed concentration of low cytotoxicity sensitized all three cell lines to 5-fluorouracil, but in the more 5-fluorouracil-sensitive SNU-C4 cell line low-dose 5-fluorouracil also added to irinotecan cytotoxicity (31).

Furthermore, while 5-fluorouracil followed by irinotecan was found to act synergistically on a human colon carcinoma cell line, this sequence did not prove better than either drug alone in a xenograft model (27). In another study (28), prior 5-fluorouracil potentiated subsequent irinotecan in a colon cancer cell line, a fact which was suggested to be due to 5-fluorouracil-stimulated DNAtopoisomerase I complex formation. The additive or synergistic effects noted by these authors with the reversed sequence were attributed to an irinotecan-induced decrease in thymidylate synthase activity (28). According to other investigators, the enhanced cytotoxicity elicited by this sequence might be attributable to $\mathrm{SN}-38$-mediated perturbation of nucleotide metabolism (29). Still other studies reported no significant effects of 5fluorouracil on topoisomerase I-mediated phenomena, or of irinotecan on thymidylate synthase activity (30).

Nevertheless, most data, including those from a number of studies not cited, indicate that irinotecan followed by 5 -fluorouracil is the most effective sequence. Using the abovementioned human colon carcinoma cell line panel, our recent data (31) conclusively showed synergistic growth inhibition when irinotecan was given before 5-fluorouracil, and antagonism with the reversed sequence. These results could be related to the introduction of more persistent DNA damage with the former sequence (31).

\section{Phase I studies}

The first clinical study with irinotecan was conducted in Japan in 1987, with the drug being administered as a 30 -min infusion (32). The dose-limiting toxicity was

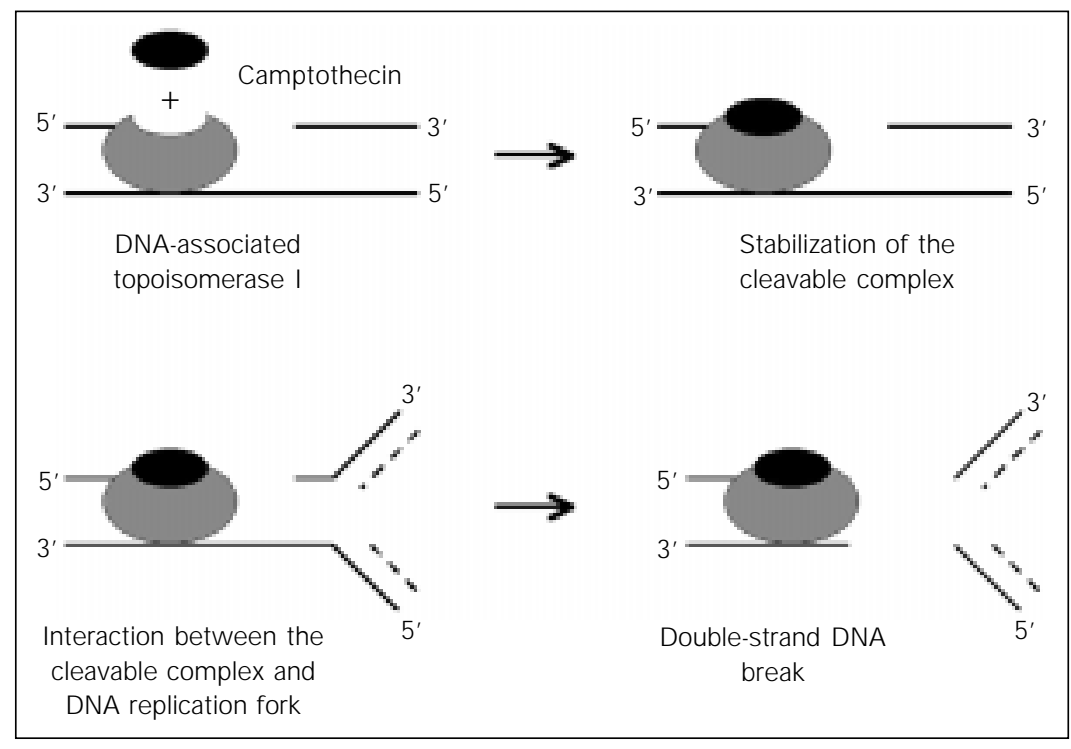

Figure 3. Mechanism of action of camptothecin. Modified from Ref. 62. 
predominantly gastrointestinal (vomiting and diarrhea). Subsequent studies in Japan and France using a variety of schedules (see for instance Refs. 22 and 24) confirmed the occurrence of both acute and delayed diarrhea, the latter more often seen in patients receiving irinotecan at repeated doses or in weekly schedules. These observations might be explained, at least in part, by the abovementioned preclinical data with laboratory mice (26), showing that irinotecan, even when given at an apparently optimum schedule, did not produce greater antitumor effects when some critical level of dose intensity was exceeded, but rather led to increased toxicity.

In addition, phase I studies revealed the occurrence of severe granulocytopenia, which was dose- and schedule-related but noncumulative. Besides these dose-limiting side effects, mild to moderate alopecia, nausea and vomiting, and fatigue were noted. The maximum tolerable dose (MTD) was $150 \mathrm{mg} \mathrm{m}^{-2}$ day $^{-1}$ in daily $\times 3$ schedules, and $145 \mathrm{mg} / \mathrm{m}^{2}$ in weekly schedules.

\section{Phase II and phase III studies}

In phase II clinical studies (see for instance Ref. 33), irinotecan produced response rates of $15-32 \%$ and $17-25 \%$ in chemotherapy-naive and pretreated patients with colorectal carcinoma, respectively. These results were consistent and indicated not only the substantial clinical efficacy of irinotecan in this disease, but also its lack of cross-resistance with 5-fluorouracil. The latter suggestion was definitely confirmed in a recent prospective, multicentric phase II study in which patients fulfilling stringent criteria for 5-fluorouracil-resistant colorectal carcinoma were treated with $350 \mathrm{mg} / \mathrm{m}^{2}$ irinotecan once every 3 weeks (33). The objective response rate among 95 evaluable patients was $13.5 \%$ (median duration of 8.5 months), and disease stabilization occurred in $44.2 \%$ of patients (median duration of 4.8 months).
Median survival was extended to 45 weeks. These and other observations raised the hope for an alternative option for patients with advanced colorectal carcinoma who relapsed after a 5-fluorouracil-based treatment. This hope was confirmed in subsequent randomized phase III clinical trials. When compared with best supportive care (33), or with best high-dose 5-fluorouracil given by infusion (33), irinotecan was demonstrated to provide significant quality of life and survival advantages (8-10 months) for patients who had failed to benefit from a prior 5-fluorouracil-based therapy, producing response rates of $14-22 \%$. An added benefit of irinotecan was its cost-effectiveness when compared to 5-fluorouracil or 5fluorouracil plus leucovorin (33).

These data led to the approval of irinotecan as second-line treatment for 5-fluorouracil-refractory advanced colorectal carcinoma in many countries. The recommended administration schedule is $125 \mathrm{mg} / \mathrm{m}^{2}$ once weekly by 90 -min infusion for 4 weeks every 6 weeks, which yields a 6-week administration intensity of $500 \mathrm{mg} / \mathrm{m}^{2}$ (33), or $350 \mathrm{mg} /$ $\mathrm{m}^{2}$ as an intravenous 90-min infusion every 3 weeks (33).

\section{Combination studies}

Many studies have been, or are being carried out to evaluate the combination of irinotecan plus 5-fluorouracil and leucovorin for its therapeutic efficacy against advanced colorectal carcinoma. Although the best schedules of drug administration have yet to be defined, the results from all these studies were encouraging in terms of response rates, time to tumor progression, and survival rates when compared to 5-fluorouracil or irinotecan alone (see for instance Refs. 34-37). Moreover, as predicted by most preclinical studies (29-31), the best results were found when irinotecan preceded 5fluorouracil.

Different schedules and sequences com- 
bining irinotecan and 5-fluorouracil have been investigated in phase I trials in Europe, the United States, and Japan (34-36). For instance, in 24 chemotherapy-naive patients treated with irinotecan followed by $24-\mathrm{h}$ continuous 5-fluorouracil plus leucovorin given once a week for 6 weeks, the MTD was established at $80 \mathrm{mg} / \mathrm{m}^{2}$ irinotecan, $2.6 \mathrm{~g} / \mathrm{m}^{2}$ 5-fluorouracil and $500 \mathrm{mg} / \mathrm{m}^{2}$ leucovorin. The dose-limiting toxicity was diarrhea, and there were 15 objective responses, including one complete response (34). In a study using a bimonthly schedule (35), 14 patients received irinotecan on day 1 , and bolus 5 fluorouracil plus leucovorin on day 2 . The MTD was $100 \mathrm{mg} / \mathrm{m}^{2}$ irinotecan, $500 \mathrm{mg} / \mathrm{m}^{2}$ leucovorin, and $2.6 \mathrm{mg} / \mathrm{m}^{2}$ 5-fluorouracil, with diarrhea as dose-limiting toxicity. The overall response rate was $45 \%$. Another phase I trial (36) used bimonthly irinotecan on day 1 , and leucovorin plus bolus 5-fluorouracil followed by 22 -h continuous infusion 5-fluorouracil on days 1 and 2 . The results from this study showed that $180-200 \mathrm{mg} / \mathrm{m}^{2}$ irinotecan could be safely combined with bolus $400 \mathrm{mg} / \mathrm{m}^{2} 5$-fluorouracil followed by continuous infusion of $600 \mathrm{mg} / \mathrm{m}^{2}$ 5-fluorouracil without causing overlapping toxicity.

In subsequent phase II studies, this combination was evaluated as second- or firstline treatment of colorectal carcinoma. In one of these studies (37), bimonthly $180 \mathrm{mg} /$ $\mathrm{m}^{2}$ irinotecan and $400 \mathrm{mg} / \mathrm{m}^{2}$ leucovorin were given on day 1 as a 90-min and a $2-\mathrm{h}$ infusion, respectively, immediately followed by a 5-fluorouracil bolus of $400 \mathrm{mg} / \mathrm{m}^{2}$ and a 46-h continuous infusion of $2.4-3 \mathrm{~g} / \mathrm{m}^{2} 5-$ fluorouracil. Of the 33 pretreated patients, 2 experienced a partial response (6\%), 20 had stabilization of their disease $(61 \%)$, and 11 progressed (33\%). Grade 3-4 toxicities occurred in 10 patients (33\%), but were manageable. Thus, this so-called FOLFIRI regimen (folinic acid, 5-fluorouracil, irinotecan) was effective as second-line therapy against metastatic colorectal carcinoma, producing acceptable toxicity.
In a recent multicenter open-label randomized trial, the combination of irinotecan plus 5-fluorouracil/leucovorin was evaluated vs 5-fluorouracil/leucovorin in patients with previously untreated disease (38). Patients were assigned to receive $180 \mathrm{mg} / \mathrm{m}^{2}$ irinotecan on day 1 , and $400 \mathrm{mg} / \mathrm{m}^{2} 5$-fluorouracil as an intravenous bolus followed by $600 \mathrm{mg} /$ $\mathrm{m}^{2}$ daily as a $22-\mathrm{h}$ continuous infusion plus leucovorin on days 1 and 2, repeated every 2 weeks; irinotecan at $80 \mathrm{mg} / \mathrm{m}^{2}$ and 5-fluorouracil at $2.3 \mathrm{~g} / \mathrm{m}^{2}$ as a $24-\mathrm{h}$ continuous infusion plus leucovorin weekly for 6 weeks, every 7 weeks, or the same regimen of 5fluorouracil/leucovorin alone. A total of 199 patients were treated with the irinotecancontaining regimen, and 188 with 5-fluorouracil/leucovorin alone. Although causing more grade 3-4 toxicities, the former combination was well tolerated when compared to the latter, producing increased response rates (49 vs 31\%), time to progression (6.7 vs 4.4 months), and survival rate (17.4 vs 14.1 months), and indicating its potential usefulness as first-line treatment of metastatic colorectal carcinoma.

Another trial (39) included 683 patients who where assigned randomly to 1 of 3 arms. Patients on one of the arms received $452 \mathrm{mg} / \mathrm{m}^{2} 5$-fluorouracil as an intravenous bolus and $20 \mathrm{mg} / \mathrm{m}^{2}$ leucovorin as an intravenous bolus daily for 5 consecutive days, every 4 weeks; irinotecan alone at $125 \mathrm{mg} /$ $\mathrm{m}^{2}$ intravenously, weekly for 4 weeks, every 6 weeks; $125 \mathrm{mg} / \mathrm{m}^{2}$ irinotecan intravenously, $500 \mathrm{mg} / \mathrm{m}^{2} 5$-fluorouracil as an intravenous bolus, and $20 \mathrm{mg} / \mathrm{m}^{2}$ leucovorin as an intravenous bolus weekly for 4 weeks, every 6 weeks. Irinotecan alone did not show any benefit compared to 5-fluorouracil/leucovorin, but the three-drug combination compared to the standard therapy increased tumor response rate (39 vs 21\%), time to disease progression (7 vs 4.3 months), and median survival time (14.8 vs 12.6 months).

These two randomized, controlled clinical trials (see Refs. 38 and 39) led to the 
approval of the irinotecan/5-fluorouracil/leucovorin regimen by the Food and Drug Administration in April, 2000, in the US. In these studies, the combination of irinotecan and 5-fluorouracil/leucovorin resulted in a significant delay in the time to tumor progression and significant improvement in survival compared with 5-fluorouracil/leucovorin alone.

\section{Oxaliplatin}

Oxaliplatin (trans- $l$-diaminocyclohexane oxalatoplatinum, L-OHP) was developed in the 1970s as one of dozens of 1,2-diaminocyclohexane $(1,2-\mathrm{DACH})$ platinum compounds in an attempt to obtain platinum analogues with a more favorable therapeutic index (40). These compounds evoked immediate enthusiasm when they appeared to exhibit no cross-resistance with cisplatin and carboplatin, and to have a preclinical activity profile distinct from that of the latter agents. However, interest in these agents waned after the occurrence of severe neurological toxicity in phase I studies with tetraplatin (or ormaplatin), the then most promising 1,2-DACH platinum compound at the time.
Clinical testing of oxaliplatin more than one decade later revealed its meaningful antitumor activity and favorable toxicity profile. Indeed, oxaliplatin proved effective in colorectal cancer both as first-line and as second-line treatment of 5-fluorouracil-refractory tumors (41). In addition, oxaliplatin was effective against both untreated and pretreated advanced ovarian carcinoma (42). Importantly, oxaliplatin does not induce the renal, hematologic, and auditory toxicity associated with cisplatin and carboplatin, its emetic effects can be effectively controlled, and its neurological toxicity - in contrast to that of most other platinum compounds - is reversible $(41,42)$

\section{Mechanism of action}

Like all platinum compounds, oxaliplatin must be converted into reactive species in displacement and aquation reactions in the bloodstream and in the intracellular environment (Figure 4). Thus, weak nucleophiles such as blood bicarbonates or intracellular dihydrogen phosphate displace the oxalate group $(40,43)$, resulting in the formation of unstable intermediates that are readily hydrolyzed to diaquated 1,2-DACH platinum.
Figure 4. Oxaliplatin biotransformation. DACH: diaminocyclohexane. Modified from Ref. 46.

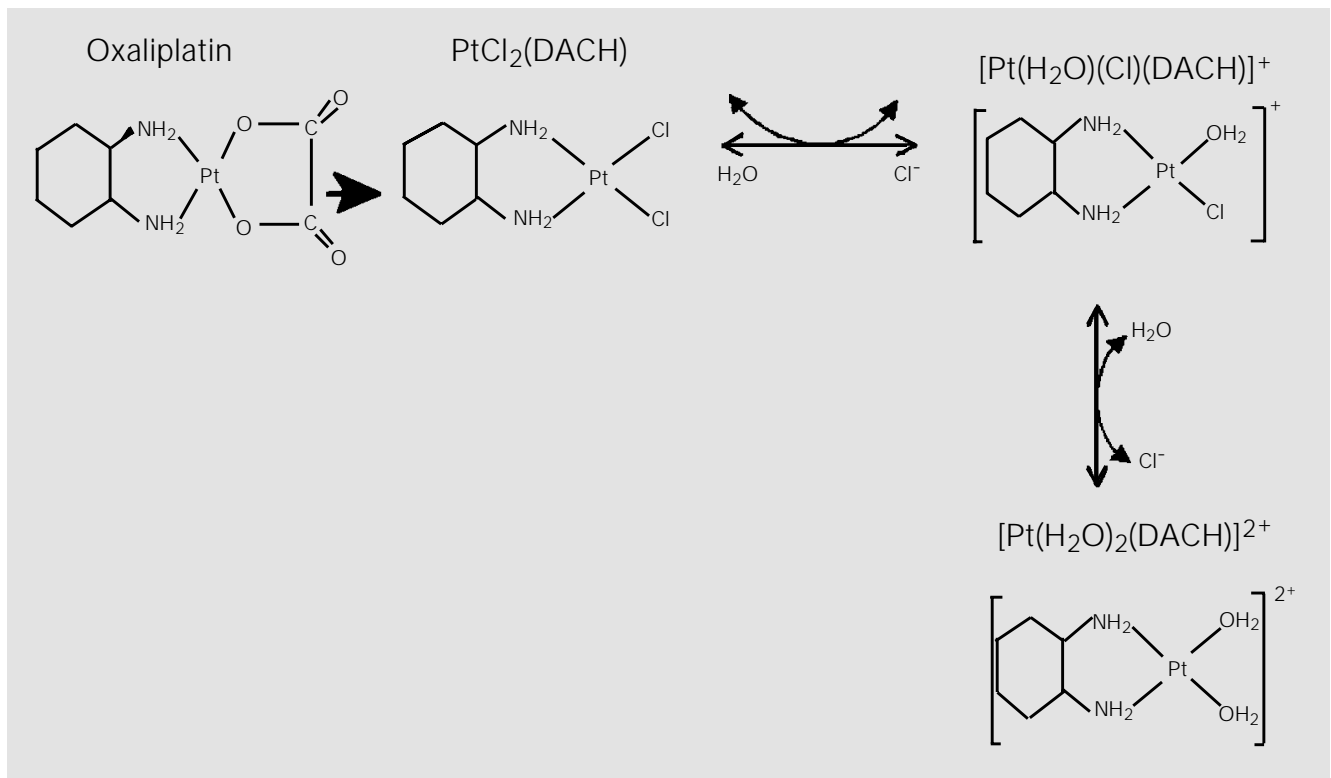


The latter species is rapidly converted into monoaqua-1,2-DACH monochloroplatinum and 1,2-DACH platinum dichloride species, which instantaneously react with DNA. As is the case with cisplatin and carboplatin, the result is the formation of platinated guanineguanine or adenine-guanine intrastrand adducts.

However, unlike the two former agents which act via a common cis-diamine intermediate, the reactive oxaliplatin intermediates retain the 1,2-DACH carrier. This has two important consequences. Firstly, the 1,2DACH ring can project into the major groove of DNA, escaping recognition by the mismatch repair enzyme complex (43). Secondly, the presence of the bulky 1,2-DACH group prevents replicative bypass, i.e., DNA synthesis past the site of the DNA adducts, as observed to occur in cisplatin-resistant cell lines (44).

These features can largely account for the requirement of fewer oxaliplatin adducts to achieve an equal level of cytotoxicity as cisplatin and carboplatin $(43,44)$, as well as for the lack of cross-resistance between the former and both latter agents $(41,42)$. The poor recognition of oxaliplatin adducts by the mismatch repair system may further explain the selective activity of oxaliplatin, unlike cisplatin and carboplatin, against tumors exhibiting mismatch repair aberrance, such as a subset of colorectal carcinomas (41). Moreover, while loss of this repair function will affect the apoptotic response of tumor cells to cisplatin and carboplatin, this is probably not the case with oxaliplatin. Consequently, platinum-responsive tumors may be more sensitive to oxaliplatin, and cisplatin-refractory tumors may respond to oxaliplatin. This presumably accounts for the above-mentioned efficacy of oxaliplatin in cisplatin-refractory patients with ovarian cancer (42).

\section{Preclinical studies}

The preclinical experience with oxali- platin has been reviewed in Ref. 45. In vitro, oxaliplatin displayed broad antitumor activity, which was in many cases equivalent to or higher than that of cisplatin. Importantly, oxaliplatin was able to inhibit the growth of cisplatin-resistant cell lines at much lower concentrations than cisplatin and carboplatin. These observations provided the first indications for the absence of cross-resistance of oxaliplatin with cisplatin and carboplatin, and its very different mechanism of cytotoxicity when compared to the latter agents (45).

These encouraging data were also seen with tumor colony-forming units isolated from patients suffering from malignant melanoma, or colon, non-small cell lung, or gastric carcinoma, including those resistant to carboplatin, 5-fluorouracil, irinotecan, paclitaxel, or doxorubicin (46). These results indicated the possibility of using oxaliplatin against a variety of drug-refractory malignancies. Results were better with protracted rather than short exposure schedules (46), a finding that may be of significance when considering administration schedules. The data from the cell culture experiments were largely confirmed in studies with laboratory animals inoculated or grafted with a variety of human tumor cells (44), in which oxaliplatin was equally effective, significantly better, or superior to cisplatin in prolonging survival (44).

Preclinical combination in vitro and in vivo studies showed additive or synergistic interactions of oxaliplatin or cisplatin with 5-fluorouracil, AG337, gemcitabine, irinotecan, paclitaxel, cisplatin, and cyclophosphamide (47). Notably, synergism between oxaliplatin and 5-fluorouracil was also seen in 5-fluorouracil- and cisplatin-resistant cell lines $(46,47)$. This phenomenon also occurred with the combination of oxaliplatin and SN-38 in the HT-29 human colon carcinoma cell line, and was attributed to SN-38induced delay of the removal of oxaliplatininduced interstrand DNA cross-links, as well 
as to an increase in the SN-38-inhibited DNA elongation in the presence of oxaliplatin adducts (48).

\section{Phase I studies}

Phase I testing (45) showed that oxaliplatin induced neither the nephrotoxicity nor the ototoxicity associated with cisplatin therapy. Emesis was in general moderate, and nausea and vomiting could effectively be controlled with antiemetics. Diarrhea was mild and was confined to a subset of patients. Hematological toxicity was much lower than that induced by equi-therapeutic doses of carboplatin, and included moderate thrombocytopenia and neutropenia.

The most consistent acute side effect was transient sensory neuropathy, which was dose-limiting and cumulative, and manifested as paresthesia and dysesthesia. These symptoms were severe with higher cumulative doses of oxaliplatin (doses higher than 1000 $\mathrm{mg} / \mathrm{m}^{2}$ have been associated with a higher than $50 \%$ toxic risk), or with combinations of oxaliplatin with other neurotoxic drugs such as taxanes and other platinum analogues (49). However, at clinically feasible dose levels of oxaliplatin, the neurological toxicity was usually moderate and completely reversible. Auditory toxicity was mild to moderate. Importantly, a few objective clinical responses were noted during these phase I studies (45).

Recommended doses for oxaliplatin monotherapy are $130 \mathrm{mg} / \mathrm{m}^{2}$ given as a 2-h intravenous infusion every 3 weeks, or 125$150 \mathrm{mg} / \mathrm{m}^{2}$ daily as a chronomodulated intravenous infusion over 5 days per week every 3 weeks.

\section{Phase II studies}

Formal phase II evaluation of oxaliplatin was only performed after several hundreds of patients had been treated with oxaliplatin in combination with 5-fluorouracil and leu- covorin $(50,51)$. These studies enrolled both pretreated and untreated patients, and used oxaliplatin-5-fluorouracil/leucovorin given as a chronomodulated continuous infusion every 3 or 2 weeks (50), or bimonthly oxaliplatin combined with high-dose continuous infusion 5-fluorouracil/leucovorin (51). Despite the occurrence of significant grade 3 and 4 gastrointestinal, bone marrow, and neurological side effects with all three regimens, and 2 toxic deaths in one study (50), objective response rates $(29-58 \%)$, median progression-free survival (6-10 months), and overall survival times were encouraging (1317.8 months), and residual metastases could be successfully excised in some patients.

Subsequent phase II evaluation of oxaliplatin alone confirmed its therapeutic efficacy against advanced colorectal carcinoma. For instance, $130 \mathrm{mg} / \mathrm{m}^{2}$ oxaliplatin every 21 days produced 9 partial responses $(24.3 \%)$ among 38 previously untreated patients with metastatic colorectal carcinoma, with a response duration of $216+$ days (52). Disease stabilization was noted in 15 patients $(40.5 \%)$, and 13 patients progressed (35.2\%). The main side effect was peripheral sensory neuropathy, but hematologic and gastrointestinal toxicities were mild. The results from two other clinical trials with $130 \mathrm{mg} / \mathrm{m}^{2}$ oxaliplatin for 21 days in chemotherapynaive patients were in agreement with those from the latter study, showing $20 \%$ objective responses and $32 \%$ stable diseases among 25 patients (53), an overall response rate of $18 \%$ among 63 patients, and a median overall survival of 13-14 months (reviewed in Ref. 54). These data suggested that oxaliplatin monotherapy may be considered as first-line treatment of advanced colorectal carcinoma.

In three phase II studies carried out on a total of 139 pretreated patients, an overall response rate of $10 \%$ and a median overall survival of 8-10 months were reported (54). These results are modest, but in the same range as those produced by other drugs that 
are active in pretreated colorectal carcinoma, indicating that oxaliplatin may also be useful for the palliation of 5-fluorouracilrefractory colorectal carcinoma.

However, oxaliplatin singly is not recommended as an option in first- or secondline treatment of advanced colorectal carcinoma, except in patients with dihydropyrimidine dehydrogenase deficiency. This condition is associated with an increased risk of very severe and potentially lethal toxicity in patients treated with 5-fluorouracil at conventional schedules, or in those with cardiotoxicity from 5-fluorouracil.

\section{Combination studies with oxaliplatin- 5-fluorouracil/leucovorin}

Studies to assess the benefits of the addition of oxaliplatin to 5-fluorouracil plus leucovorin were initiated following the observation that this combination acted synergistically in in vitro as well as in vivo murine models (45). These studies enrolled chemotherapy-naive and 5-fluorouracil-pretreated patients and were performed according to various trial designs, viz. as one-armed phase II trials, two-armed phase III trials, and compassionate-use programs.

The results from these studies were comparable in terms of the efficacy and tolerability of the combination of 5-fluorouracil/leucovorin with oxaliplatin. Response rates, progression-free survival, and overall survival were consistently and significantly higher with oxaliplatin plus 5-fluorouracil/leucovorin when compared with 5-fluorouracil/ leucovorin alone. The main problem with the addition of oxaliplatin to 5-fluorouracil/ leucovorin was diarrhea, which was probably related to a cumulative effect of both drugs, but could be managed. These data led to proposals to use the oxaliplatin-5-fluorouracil/leucovorin combination as first- or second-line treatment against advanced colorectal carcinoma, as well as against liver metastases associated with the disease.
Support for the efficacy of the combination as a first-line treatment option came from a recent study (55) in which 200 patients were randomly assigned to receive a 5day course of chronomodulated 5-fluorouracil and leucovorin (700 and $300 \mathrm{mg} / \mathrm{m}^{2}$ daily, respectively) with or without oxaliplatin (125 $\mathrm{mg} / \mathrm{m}^{2}$ ) on the first day of each course. The treatment was repeated every 21 days. The most severe toxicity was grade 3-4 diarrhea in $43 \%$ of patients given oxaliplatin. Median progression-free survival time was slightly higher in this group when compared to that receiving 5-fluorouracil/leucovorin alone (8.7 versus 6.1 months), and there were no significant differences in median survival time between the two arms (19.4 and 19.9 months). However, objective response rates were $53 \%$ in the former group versus only $16 \%$ in the latter.

Indications for the applicability of oxaliplatin-5-fluorouracil/leucovorin as secondor third-line treatment of colorectal carcinoma came from various studies evaluating this combination in patients with metastatic colorectal carcinoma who were refractory to 5-fluorouracil and/or irinotecan, or who progressed while on a second 5-fluorouracilbased therapy (56). These studies used weekly or bimonthly schedules, bolus and/ or continuous infusion 5-fluorouracil at conventional or at high doses, and/or oxaliplatin at full dose or at $50 \%$ reduced doses, and were carried out according to one-armed or two-armed designs. The results from these studies showed that the oxaliplatin-5-fluorouracil/leucovorin combination could effectively palliate treatment-refractory colorectal carcinoma, producing meaningful objective response rates, a considerable number of cases of disease stabilization, and significant improvements in time to progression and survival. Side effects were occasionally severe, consisting of grade 3 and 4 bone marrow, gastrointestinal, and/or neurological toxicity, which was, however, manageable. 
These results justified the employment of this combination in compassionate-use programs. In one such study (57), $80-100 \mathrm{mg} / \mathrm{m}^{2}$ oxaliplatin every 2 weeks or $100-135 \mathrm{mg} / \mathrm{m}^{2}$ every 3 weeks added to 5 -fluorouracil plus leucovorin produced an overall response rate of $25.5 \%$, a median time to progression of 4.1 months, and a median overall survival of 9.6 months in 98 evaluable patients with advanced colorectal carcinoma.

Another important therapeutic advantage of the oxaliplatin-5-fluorouracil/leucovorin combination is its capacity to shrink hepatic metastases in a significant number of patients with metastatic colon carcinoma $(55,57)$. This led not only to improvement in two- and five-year survival rates (55), but also to an increased number of successes in subsequent attempts involving secondary potentially curative surgery $(55,57)$.

An important phase III study (58) investigated the effect of combining oxaliplatin with 5-fluorouracil/leucovorin, with progression-free survival as the primary end point. For that purpose 420 previously untreated patients with measurable disease were randomized to receive a 2-h infusion of leucovorin, $200 \mathrm{mg} / \mathrm{m}^{2}$ daily, followed by $400 \mathrm{mg} /$ $\mathrm{m}^{2}$ 5-fluorouracil as bolus daily, and 22-h infusion of $600 \mathrm{mg} / \mathrm{m}^{2}$ daily for 2 consecutive days, every 2 weeks, either alone or together with $85 \mathrm{mg} / \mathrm{m}^{2}$ oxaliplatin as a 2-h infusion on day 1. Patients allocated to oxaliplatin plus 5-fluorouracil/leucovorin had significantly longer progression-free survival (9.0 vs 6.2 months) and a better response rate (50.7 vs 22.3\%) when compared with the control arm. The improvement in overall survival did not reach significance (16.2 vs 14.7 months).

The combination of oxaliplatin with 5fluorouracil/leucovorin seems beneficial as first-line therapy in advanced colorectal cancer. However, no improvement in terms of overall survival was obtained $(55,58)$. These results led to disapproval of this therapy by the Food and Drug Administration in the USA.

\section{Combination studies with oxaliplatin-irinotecan}

Four phase I/II trials with oxaliplatin followed one hour later by irinotecan have been carried out on patients with 5-fluorouracilrefractory gastrointestinal carcinomas, including colorectal carcinoma $(59,60)$. Two studies evaluated this combination using a 3week schedule (59), two used a 2 -week schedule (reviewed in Ref. 60). The recommended doses for oxaliplatin and irinotecan for the 3 week schedule were 85 and $200 \mathrm{mg} / \mathrm{m}^{2}$, respectively, and for the 2 -week schedule 85 and $175 \mathrm{mg} / \mathrm{m}^{2}$, respectively. Despite the occurrence of grade 3 and 4 gastrointestinal, bone marrow, and neurological toxicities, objective response rates were encouraging $(34 \%)$, suggesting that this combination is feasible and active in advanced colorectal carcinoma.

Various other schedules are being explored in additional studies (reviewed in Ref. 60). For instance, in a phase II trial the combination of $85 \mathrm{mg} / \mathrm{m}^{2}$ oxaliplatin on days 1 and 15 plus $80 \mathrm{mg} / \mathrm{m}^{2}$ irinotecan on days 1 , 8 , and 15 , given every 4 weeks with or without granulocyte colony-stimulating factor, produced 2 complete responses $(6 \%)$ and 13 cases of disease stabilization $(36 \%)$ in 36 patients with metastatic colorectal carcinoma who had been pretreated with 5fluorouracil/leucovorin. There were 7 cases of grade 3-4 granulocytopenia, and gastrointestinal toxicity (nausea, vomiting, and diarrhea) was dose-limiting (60).

\section{Concluding remarks and future prospects}

Today, advanced colorectal cancer is still an incurable disease. Although successful surgery is possible in selected patients, current treatment modalities are still insuffi- 
ciently efficacious. This also holds true for chemotherapy that has thus far only palliative value. However, the availability of a number of new 5-fluorouracil analogues, new folate antagonists, as well as novel, effective cytotoxic agents such as irinotecan and oxaliplatin, is generally considered to have a significant impact on the treatment of colorectal carcinoma. Clinical trials have already established the efficacy and tolerability of these agents singly, which led to their recommended use as second-line treatment in 5-fluorouracil-refractory disease, or in patients for whom 5-fluorouracil is contraindicated. Other studies showed that their addition to the classical 5-fluorouracil/leucovorin combination significantly increases response rates, time to disease progression, and survival. The challenge is now to fully exploit the opportunities at hand by designing effective multidrug regimens. This will require studies to optimize dosing, timing, and schedule of administration of each agent, to define the roles of the new 5-fluorouracil analogues and folate antagonists in cancer therapy with respect to those of the parent compounds, to determine optimum combination(s) of the most active drugs, and to establish optimum drug administration sequences. The results from these studies will contribute to the design of more effective chemotherapy treatment protocols for advanced colorectal carcinoma, both as firstand second-line treatment, and in the adjuvant setting.

In addition, current knowledge on the predictive value of thymidylate synthase and dihydropyrimidine dehydrogenase (7) expression will enable us to select patients for potential effective treatment. Patients with a high intratumoral level of either thymidylate synthase or dihydropyrimidine dehydrogenase should not receive 5-fluorouracil-based treatment, unless a dihydropyrimidine dehydrogenase inhibitor is included. Patients with a high thymidylate synthase should receive an irinotecan or an oxaliplatin-based treatment schedule. These prospective studies will permit us to tailor treatment to each patient and to give them the most active treatment modality.

\section{References}

1. Parker SL, Tong $\mathrm{T}$, Bolden S \& Wingo PA (1996). Cancer statistics, 1996. CA: A Cancer J oumal for Clinicians, 46: 5-27.

2. Ministério da Saúde. Instituto Nacional de Câncer (INCA) (1999). Coordenação de Programas de Controle de Câncer - ProOnco. O Problema do Câncer no Brasil. 4th edn. Rio de J aneiro.

3. Cohen AM, Minsky BB \& Schilsky RL (1997). Cancer of the colon. In: DeVita VT, Hellman S \& Rosenberg AS (Editors), Cancer: Principles and Practice of Oncology. Lippincott-Raven, Philadelphia.

4. Grem J L (1996). Fluoropyrimidines. In: Chabner BA \& Longo DL (Editors), Cancer Chemotherapy and Biotherapy: Principles and Practice. Lippincott-Raven, Philadelphia.

5. Pinedo HM \& Peters GJ (1988). Fluorouracil: biochemistry and pharmacology. J ournal of Clinical Oncology, 6: 16531664.
6. Santi DV, McHenry CS \& Sommer H (1974). Mechanism of interaction of thymidylate synthase with 5-fluorodeoxyuridylate. Biochemistry, 13: 471-481.

7. Van Triest B \& Peters GJ (1999). Thymidylate synthase: a target for combination therapy and determinant of chemotherapeutic response in colorectal cancer. Oncology, 57: 179-194.

8. Mandel HG (1981). The target cell determinants of the antitumor actions of 5-FU: does 5-FU incorporation into RNA play a role. Cancer Treatment Reports, 65: 6371.

9. Schuetz J D \& Diasio RB (1985). The effect of 5-fluorouracil on DNA chain elongation in intact bone marrow cells. Biochemical and Biophysical Research Communications, 133: 161-167.

10. Meta-analysis Group in Cancer (1998). Efficacy of intravenous continuous infusion of fluorouracil compared with bolus ad- ministration in advanced colorectal cancer. J ournal of Clinical Oncology, 16: 301308.

11. Kemeny N, Huang $Y$, Cohen AM, Shi W, Conti J A, Brennan MF, Bertino J R, Tumbull $A D$, Sullivan $D$, Stockman J , Blumgart LH \& Fong Y (1999). Hepatic arterial infusion of chemotherapy after resection of hepatic metastases from colorectal cancer. New England J ournal of Medicine, 341: 2039-2048.

12. Peters GJ \& Van Groeningen CJ (1991). Clinical relevance of biochemical modulation of 5-fluorouracil. Annals of Oncology, 2: 469-480.

13. Advanced Colorectal Cancer Meta-analysis Project (1992). Modulation of fluorouracil by leucovorin in patients with advanced colorectal cancer: evidence in terms of response rate. J ournal of Clinical Oncology, 10: 896-903.

14. Cassidy J (1999). Potential of Xeloda ${ }^{\circledR}$ in 
colorectal cancer and other solid tumors. Oncology, 57: 27-32.

15. Hoff PM, Royce M, Medgyesy D, Brito R \& Pazdur R (1999). Oral fluoropyrimidines. Seminars in Oncology, 26: 640-646.

16. Danenberg PV, Malli $\mathrm{H} \&$ Swenson S (1999). Thymidylate synthase inhibitors. Seminars in Oncology, 26: 621-631.

17. Wall $M$, Wani $M C$, Cook CE, Palmar $\mathrm{KH}$, McPhail AT \& Sim GA (1966). Plant antitumor agents: I. The isolation and structure of camptothecin, a novel alkaloidal leukemia and tumor inhibitor from Camptotheca acuminata. J oumal of the American Chemical Society, 88: 3888-3890.

18. Moertel CG, Schutt AJ, Reitemeier RJ \& Hahn RG (1972). Phase II study of camptothecin (NSC-100800) in the treatment of advanced gastrointestinal cancer. Cancer Chemotherapy Reports, 56: 95-101.

19. Hsiang Y-H \& Liu LF (1988). Identification of mammalian topoisomerase I as an intracellular target of the anticancer drug camptothecin. Cancer Research, 48: 1722-1726.

20. Takimoto $\mathrm{CH}$, Kieffer LV \& Arbuck SG (1997). DNA topoisomerase I inhibitors. Cancer Chemotherapy and Biological Response Modifiers,17: 80-113.

21. Bleiberg H (1999). CPT-11 in gastrointestinal cancer. European J ournal of Cancer, 35: 371-379.

22. Sandler A \& van Oosterom AT (1999). Irinotecan in cancers of the lung and cervix. Anticancer Drugs, 10 (Suppl 1): S13S17.

23. Friedman HS, Petros WP, Friedman $A H$, Schaaf LJ, Kerby T, Lawyer J, Parry M, Houghton PJ, Lovell S, Rasheed K, Cloughsey T, Stewart ES, Colvin OM, Provenzale JM, McLendon RE, Bigner DD, Cokgor I, Haglund M, Rich J , Ashley D, Malczyn J, Elfring GL \& Miller LL (1999). Irinotecan therapy in adults with recurrent or progressive malignat glioma. J ournal of Clinical Oncology, 17: 15161525.

24. Slichenmyer WJ, Rowinsky EK, Donehower RC \& Kaufmann SH (1993). The current status of camptothecin analogues as antitumor agents. J ournal of the National Cancer Institute, 85: 271-291.

25. Rivory LP, Bowles R, Robert J \& Pond SM (1996). Conversion of ininotecan (CPT-11) to its active metabolite, 7-ethyl-10-hydroxycamptothecin (SN-38), by human liver carboxylesterase. Biochemical Pharmacology, 52: 1103-1111.

26. Houghton PJ, Stewart CF, Zamboni WC, Thompson J , Luo X, Danks MK \& Houghton J A (1996). Schedule-dependent effi- cacy of camptothecins in models of human cancer. Annals of the New York Academy of Sciences, 203: 188-201.

27. Guichard S, Cussac D, Hennebelle I, Bugat R \& Canal P (1997). Sequence-dependent activity of the irinotecan-5-FU combination in human colon-cancer model HT-29 in vitro and in vivo. International J oumal of Cancer, 73: 729-734.

28. Guichard S, Hennebelle I, Bugat R \& Canal $P$ (1998). Cellular interactions of 5fluorouracil and the camptothecin analogue CPT-11 (irinotecan) in a human colorectal carcinoma cell line. Biochemical Pharmacology, 55: 667-676.

29. Mullany $\mathrm{S}$, Svingen $\mathrm{PA}$, Kaufman $\mathrm{SH} \&$ Erlichmann C (1998). Effect of adding the topoisomerase I poison 7-ethyl-10-hydroxycamptothecin (SN-38) to 5-fluorouracil and folinic acid in HCT-8 cells - elevated DTTP pools and enhanced cytotoxicity. Cancer Chemotherapy and Pharmacology, 42: 391-399.

30. Pavillard $V$, Formento $P$, Rostagno $P$, Formento J L, Fischel J L, Francoual $M$, Etienne MC \& Milano G (1998). Combination of ininotecan (CPT-11) and 5-fluorouracil with an analysis of cellular determinants of drug activity. Biochemical Pharmacology, 56: 1315-1322.

31. Mans DRA, Grivicich I, Peters GJ \& Schwartsmann G (1999). Sequence-dependent growth inhibition and DNA damage formation by the irinotecan-5-fluorouracil combination in human colon carcinoma cell lines. European J ournal of Cancer, 35: 1851-1861.

32. Taguchi $T$, Wakui $A$, Hasegawa $K$, Niitani H, Furue H, Ohta K \& Hattori T (1990). Phase I clinical study of CPT-11. J apanese J oumal of Cancer Chemotherapy, 17: 115120.

33. Vanhoefer $U$, Harstrick $A$, Achterrath $W$, Cao S, Seeber S \& Rustum YM (2001). Irinotecan in the treatment of colorectal cancer: clinical overview. J ournal of Clinical Oncology, 19: 1501-1518.

34. Comella P, Casaretti R, De Vita F, Avallone A, Orditura M, Petrillo A, Gravina A, Faranda A, Comis S, Comella G \& Catalano G (1999). Concurrent irinotecan and 5-fluorouracil plus leuvo-folinic acid given every other week in the first-line management of advanced colorectal carcinoma: a phase I study of the Southern Italy Cooperative Oncology Group. Annals of Oncology, 10: 915-921.

35. Ducreux M, Ychou M, Seitz JF, Bonnay $M$, Bexon A, Armand JP, Mahjoubi M, Mery-Mignard D \& Rougier P (1999). Irinotecan combined with bolus fluorouracil, continuous infusion fluorouracil, and highdose leucovorin every two weeks (LV5FU2 regimen): a clinical dose-finding and pharmacokinetic study in patients with pretreated metastatic colorectal cancer. J ournal of Clinical Oncology, 17: 2901-2908.

36. Vanhoefer $\mathrm{U}$, Harstrick $\mathrm{A}$, Köhne $\mathrm{C}-\mathrm{H}$ Müller C, Kretschmar A, Kraub C, Wilke H \& Seeber S (1999). Phase I study of a weekly schedule of ininotecan, high-dose leucovorin, and infusional fluorouracil as first-line chemotherapy in patients with advanced colorectal cancer. J ournal of Clinical Oncology, 17: 907-913.

37. André $T$, Louvet $C$, Maindrault-Goebel $F$, Couteau C, Mabro M, Lotz J P, Gilles-Amar V, Krulik M, Carola E, Izrael V \& de Gramont A (1999). CPT-11 (irinotecan) addition to bimonthly, high-dose leucovorin and bolus and continuous-infusion 5-fluorouracil (FOLFIRI) for pretreated metastatic colorectal cancer. European J ournal of Cancer, 35: 1343-1347.

38. Douillard JY, Cunningham D, Roth $A D$, Navarro M, J ames RD, Karasek P, J andik $P$, Iveson T, Carmichael J , Alakl M, Gruia G, Awad L \& Rougier P (2000). Irinotecan combined with fluorouracil compared with fluorouracil alone as first-line treatment for metastatic colorectal cancer: a multicentre randomised trial. Lancet, 355: 1041-1047.

39. Saltz LB, Cox J V, Blanke C, Rosen LS, Fehrenbacher L, Moore MJ, Maroun J A, Ackland SP, Locker PK, Pirotta N, Elfring GL \& Miller LL (2000). Irinotecan plus fluorouracil and leucovorin for metastatic colorectal cancer. Irinotecan Study Group. New England J oumal of Medicine, 343: 905-914.

40. Cvitkovic E (1998). A historical perspective on oxaliplatin: rethinking the role of platinum compounds and learning from near misses. Seminars in Oncology, 25 (Suppl 5): 1-3.

41. Machover D, Diaz-Rubio E, de Gramont A, Schilf A, Gastiaburu J J, Brienza S, Itzhaki M, Metzger G, N'Daw D, Vignoud J , Abad A, Francois E, Gamelin E, Marty M, Sastre J , Seitz J F \& Ychou M (1996). Two consecutive phase II studies of oxaliplatin (L$\mathrm{OHP}$ ) for treatment of patients with advanced colorectal carcinoma who were resistant to previous treatment with fluoropyrimidines. Annals of Oncology, 7: 9598.

42. Chollet $P$, Bensmaine MA, Brienza $S$, Deloche C, Cure H, Caillet H \& Cvitkovic E (1996). Single agent activity of oxaliplatin in heavily pretreated advanced epithelial 
ovarian cancer. Annals of Oncology, 7: 1065-1070.

43. Fink $D$, Nebel $S$, Aebi $S$, Zheng $H$, Cenni B, Nehme A, Christen RD \& Howell SB (1996). The role of mismatch repair in platinum drug resistance. Cancer Research, 56: 4881-4886.

44. Mamenta EL, Poma EE, Kaufmann WK, Delmastro DA, Grady HL \& Chaney SG (1994). Enhanced replicative bypass of platinum-DNA adducts in cisplatin-resistant human ovarian carcinoma cell lines. Cancer Research, 54: 3500-3505.

45. Raymond E, Chaney SG, Taamma A \& Cvitkovic E (1998). Oxaliplatin: A review of preclinical and clinical studies. Annals of Oncology, 9: 1053-1071.

46. Raymond E, Lawrence R, Izbicka E, Faivre S \& Von Hoff DD (1998). Activity of oxaliplatin against human tumor colony forming units. Clinical Cancer Research, 4: 1021-1029.

47. Raymond E, Djelloul C, Buquet-Fagot F, Mester E \& Gespach C (1996). Synergy between the non-classical thymidase synthase inhibitor AG337 (Thymitaq) and cisplatin in human colon and ovarian cancer cells. Anticancer Drugs, 7: 752-757.

48. Zeghari-Squalli N, Raymond E, Cvitkovic $E$ \& Goldwasser F (1999). Cellular pharmacology of the combination of the DNA topoisomerase I inhibitor SN38 and the diaminocyclohexane platinum derivative oxaliplatin. Clinical Cancer Research, 5: 1189-1196.

49. Extra J M, Marty M, Brienza S \& M isset J L (1998). Pharmacokinetics and safety profile of oxaliplatin. Seminars in Oncology, 25 (Suppl 5): 13-22.

50. Levi $F$, Misset J L, Brienza $S$, Adam $R$, Metzger G, Itzakhi M, Caussanel JP, Kunstlinger $F$, Lecouturier $S \&$ DescorpsDeclere A (1992). A chronopharmacologic phase II clinical trial with 5-fluorouracil, folinic acid, and oxaliplatin using an ambulatory multichannel programmable pump: High antitumor effectiveness against metastatic colorectal cancer. Cancer, 69: 893-900.

51. de Gramont A, Vignoud J, Toumigand C, Louvet C, Andre T, Varette C, Raymond E, Moreau S, Le Bail N \& Krulik M (1997). Oxaliplatin with high dose folinic acid and 5-fluorouracil 48-h infusion in pretreated metastatic colorectal cancer. European J oumal of Cancer, 33: 214-219.

52. Becouarn $Y$, Ychou $M$, Ducreux $M$, Borel C, Bertheault-Cvitkovic F, Seitz J F, Nasca S, Nguyen TD, Paillot B, Raoul J L, Duffour J , Fandi A, Dupont-Andre $G \&$ Rougier $P$ (1998). Phase II trial of oxaliplatin as firstline chemotherapy in metastatic colorectal cancer patients. J oumal of Clinical Oncology, 16: 2739-2744.

53. Diaz-Rubio E, Sastre J, Zaniboni A, Labianca $\mathrm{R}$, Cortes-Funes $\mathrm{H}$, de Braud $\mathrm{F}$, Boni $C$, Benavides $M$, Dallavalle $G \&$ Homerin M (1998). Oxaliplatin as single agent in previously untreated colorectal carcinoma patients: a phase II multicentric study. Annals of Oncology, 9: 105-108.

54. Becouarn $Y \& \&$ Rougier P (1998). Clinical efficacy of oxaliplatin monotherapy: phase II trials in advanced colorectal cancer. Seminars in Oncology, 25 (Suppl 5): 2331.

55. Giacchetti S, Perpoint B, Zidani R, Le Bail $\mathrm{N}$, Faggiuolo R, Focan C, Chollet P, Llory J F, Letoumeau Y, Coudert B, BertheaultCvitkovic F, Larregain-Foumier D, Le Rol A, Walter S, Adam R, Misset J L \& Levi F (2000). Phase III multicenter randomized trial of oxaliplatin added to chronomodulated fluorouracil-leucovorin as first-line treatment of metastatic colorectal cancer. J oumal of Clinical Oncology, 18: 136-147.

56. J aninis J, Papakostas P, Samelis G, Skarlos D, Papagianopoulos P \& Fountzilas G
(2000). Second-line chemotherapy with weekly oxaliplatin and high-dose 5-fluorouracil with folinic acid in metastatic colorectal carcinoma: a Hellenic Cooperative Oncology Group (HeCOG) phase II feasibility study. Annals of Oncology, 11: 163-167.

57. MacDonald J S \& Astrow AB (2001). Adjuvant therapy of colon cancer. Seminars in Oncology, 28: 30-40.

58. de Gramont A, Figer A, Seymour M Homerin M, Hmissi A, Cassidy J, Boni C, Cortes-Funes $\mathrm{H}$, Cervantes A, Freyer G, Papamichael D, Le Bail N, Louvet C, Hendler D, de Braud F, Wilson C, Morvan $F \&$ Bonetti A (2000). Leucovorin and fluorouracil with or without oxaliplatin as firstline treatment in advanced colorectal cancer. J ournal of Clinical Oncology, 18: 2938-2947.

59. Goldwasser F, Gross-Goupil M, Tigaud J M, Di Palma M, Marceau-Suissa J, Wasserman E, Yovine A, Misset JL \& Cvitkovic E (2000). Dose escalation of CPT-11 in combination with oxaliplatin using an every two weeks schedule: a phase I study in advanced gastrointestinal cancer patients. Annals of Oncology, 11: 1463-1470.

60. Pelley RJ (2001). Oxaliplatin: a new agent for colorectal cancer. Current Oncology Reports, 3: 147-155.

61. Peters GJ \& J ansen G (1996). Resistance to antimetabolites. In: Schilsky RL, Milano GA \& Ratain MJ (Editors), Principles of Antineoplastic Drug Development and Pharmacology. Marcel Dekker, Inc., New York.

62. Takimoto $\mathrm{CH} \&$ Arbuck SG (1996). Camptothecins. In: Chabner BA \& Longo DL (Editors), Cancer Chemotherapy and Biotherapy: Principles and Practice. Lippincott-Raven, Philadelphia. 